\title{
Recommender System for Engineering College
}

\author{
Meshwa Mistry, Vidhi Suthar, KaPatel Shruti, Priyang Bhatt
}

\begin{abstract}
In this paper, we present research on developing a recommender system that helps students who want to take admission in engineering colleges. There are various engineering colleges in Gujarat. After completion of $12^{\text {th }}$ Science, if students want to seek their admission in engineering, there are so many choices. Students generally face problems in choosing the better college as per their merit number. Colleges may have facilities such as campus facilities, university grants, the infrastructure of institutes, hostel facility, NBA and NAAC grading, placement record, tie-up with industries, faculties or educational history too. Students and parents do not have exact information about these all. Moreover, there is no such website or an application which gives the suggestion or recommend institutions where students can get admission. After studying these issues facing by parents and students, we are going to develop a recommender system for engineering institutes which can recommend to students as per their merit number and user reviews.
\end{abstract}

Keywords: Recommendation System, SVD, Pivot table Similarity Measure

\section{INTRODUCTION}

$\mathrm{N}_{\mathrm{o}}$ owadays there are many websites available on the internet that give knowledge about various engineering colleges, so students get confused about which information is proper for taking admission to college. After completion of $12^{\text {th }}$ Science lot of students try to get admission in engineering college. Colleges may have facilities such as campus facilities, university grants, the infrastructure of institutes, hostel facility, NBA and NAAC grading, placement record, tie-up with industries, faculties or educational history too. some of the students succeed to get admission into their desired college. But students from small cities and villages have less knowledge about various engineering colleges in Gujarat. If these students will have sufficient knowledge then the students take admission as per their interest. Due to this problem, we are going to develop a system which helps them to select better institution based on various factors and parameters. We went through various websites and forums and noted the various problems faced by users and their needs and requirements for our problem domain. The main objective of our system is to recommend engineering institutions based on past engineering admission records, the general facilities provided by institutions and user and alumni reviews.

Revised Manuscript Received on May 20, 2020.

Meshwa Mistry, Department of Computer Engineering, G H Patel College of Engineering \& Technology, Vallabh Vidyanagar, Gujarat, India.

Vidhi Suthar, Department of Computer Engineering, G H Patel College of Engineering \& Technology, Vallabh Vidyanagar, Gujarat, India.

KaPatel Shruti, Department of Computer Engineering, G H Patel College of Engineering \& Technology, Vallabh Vidyanagar, Gujarat, India.

Priyang Bhatt, Assistant Professor, Department of Computer Engineering, G H Patel College of Engineering \& Technology, Vallabh Vidyanagar, Gujarat, India.

Now a day's recommender system is used in many areas. Recommender System is an information filtering technique, which provides users with information, which he/she may be interested in. A recommender system or a recommendation system is a subclass of an information filtering system that seeks to predict the "rating" or "preference" a user would give to an item. A Recommender System is a computer program that helps a user to discover a product and contain by predicting the user rating of each item and showing them the item, they would rate highly. With online shopping, consumers have nearly infinite choices. No one has enough time to try every product for a sale. The Recommender Systems play an important role in helping users to find products in contain they only care about.

Various types of recommendation system:

Collaborative filtering

Content-Based Filtering

Hybrid Recommendation Systems

\section{Content-Based Filtering}

These filtering methods are based on the description of a product. The recommended products are similar to the ones that a user has liked in the past. For example, there is one article that user already read in the past, and in future user wants to read another article so the system recommends based on what a user read in the past.

\section{Collaborative Filtering}

Collaborative filtering systems make recommendations only based on how users rated products in the past, not based on anything about the products themselves. In collaborative filtering, the recommendation system does not know the actual product it is recommending. It only knows how other users rated the product. For example, if a person $\mathrm{A}$ likes items 1,2,3 and B like 2,3,4 then they have similar interests and A should like items 4 and B should like item 1. Our system falls under item-based collaborative filtering. There are two types of collaborative filtering..

\section{User-User collaborative filtering}

This algorithm first finds the similarity score between users. Based on this similarity score, it then picks out the most similar users and recommends products that these similar users have liked or bought previously.

The prediction $P u, i$ is given by:

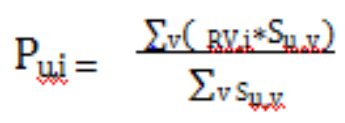

Here,

- $P u, i$ is the prediction of an item

- $\quad R v, i$ is the rating given by a user $v$ to a movie $i$

- $S u, v$ is the similarity between users 


\section{Recommender System for Engineering College}

\section{Item-Item collaborative filtering}

In this algorithm, we compute the similarity between each pair of items So in our case we will find the similarity between each college pair and based on that we will recommend similar colleges that are liked by the users in the past. This algorithm works similarly to user-user collaborative filtering with just a little change - instead of taking the weighted sum of ratings of "user-neighbors", we take the weighted sum of ratings of "item-neighbors". The prediction is given by:

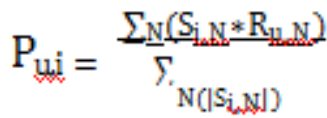

Now we will find the similarity between items.

$$
\operatorname{sim}(i, j)=\cos (\vec{i}, \vec{j})=\frac{\vec{i} \cdot \vec{j}}{\|\vec{i}\|_{2} *\|\vec{j}\|_{2}}
$$

Now, as we have the similarity between each college and the ratings, predictions are made and based on those predictions, similar colleges are recommended.

\section{Hybrid Filtering}

In a hybrid recommendation system combining collaborative and content-based recommendations can be more effective. Hybrid approaches can be implemented by making content-based and collaborative-based predictions separately and then combining them.

\section{LITERATURE SURVEY}

The today's recommendation system is used in many areas to provide ease of selection of product or item or predict the user needs but there is no such system that can predict the college not based on the marks but the best match according to the student profile. This system provides a ranked list of colleges or universities along with all government courses available to the student according to the preference given by the student. Parameters that are used for ranking the colleges from Alumni are College Environment, Faculty, Placement, Culture, Campus, etc. [1]

The System gathers data about Users from social media and crawls College data from trusted sites. For getting an information seeker's interest explicit profiling approach is been used. Recommendation Engine collects User data from Social media and builds User profile based on various parameters like Faculty, Campus life, Placements, etc. likewise, it takes into consideration Alumni or current student data into consideration for building College Profile. Based on User \& College Profiling recommendations are generated for information seekers using a hybrid approach.[1]

In this paper, we presented the design and implementation of Friend book, a semantic-based friend recommendation system for social networks. Different from the friend recommendation mechanisms relying on social graphs in existing social networking services, Friend book extracted lifestyles from user- centric data collected from sensors on the Smartphone and recommended potential friends to users if they share similar lifestyles.[2]

In SVD they used ALSWR (Alternating-Least- Squares with Weighted- $\lambda$-Regularization) factorize recommendation. The values required for factorization are the data model, number

of features, value of lambda, and number of iterations. ALSWR is an iterative algorithm to solve the low-rank factorization. The values of the recommended changes as the value of lambda changes and several iterations changes. Lambda is the regularization parameter that is used to prevent over-fitting. For the User-College Matrix, similarity measure defines the association between the two. But this measure and in turn recommendations are controlled by Number of iterations and Lambda value.[1]

In this paper, applied research on designing and developing a recommender system for graduate admission seekers which can help them to choose graduate school matching their entire academic profile. Here developed a technique to transform relational database for students' all types of relevant information into a universal database format using academic data of successful students who have already got the opportunity to study abroad. After they developed an algorithm for grad school recommender system which can calculate the similarity between training and test data set based on weighted scores using mean squared deviation similarity metrics. They used the K-nearest Neighbor algorithm for calculating top $\mathrm{N}$ similar users for the test users and recommend Top $\mathrm{K}$ universities to users from $\mathrm{N}$ similar users. Finally, the recommender system will recommend a list of universities to apply for graduate admission to pursue higher study abroad with funding.[3]

Calculate a weighted score from prior information of successful applicants such as undergrad CGPA, GRE, TOFEL_IELTS Score, Job_experience, Research_experience, Research_area \& Intended_Outgoing_Country, intended_Semester, Intended_admission_program. Calculate the similarity between the user's scores using the mean squared deviation

\begin{tabular}{|c|c|c|c|c|c|c|c|c|c|c|}
\hline Email Adovess & Insitutename & Infrastuc h & fifaspoots & & mouslif & $\mathrm{SP}$ & & $\mathrm{M} / \mathrm{Cl}$ & & all Grade \\
\hline pareknnaitik139gmail.com & A.D.Patel Institute Of Tech, Karamsad & 5 & 7 & 6 & 6 & 0 & 4 & 5 & ${ }^{4}$ & 4 \\
\hline bhargavatele3979gegnail.co & 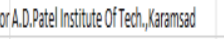 & 9 & & 6 & 10 & 10 & 7 & 8 & 8 & 8 \\
\hline eepresesheaditacin & A.D.Patel | Institute Of Tech, Karamssod & 10 & 9 & 10 & 10 & 9 & 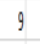 & 9 & 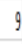 & J \\
\hline 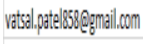 & 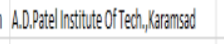 & 10 & 6 & 10 & 10 & 10 & 10 & 10 & 5 & 5 \\
\hline dapokiva@genail.com & A.D.Pagtel Institute Of Tech,_(Bramssod & 9 & 9 & 0 & 9 & 6 & 0 & 5 & & 0 \\
\hline svisovalia@egmail.com & A.D.PBgtel lnstitute Of Tech, Karemsad & 8 & 5 & 9 & 8 & 5 & 8 & 7 & 6 & 6 \\
\hline cuddani7@egmail.com & A.D.P.Patel lnstitute OT Tech, (Bramsad & 9 & 7 & 10 & 7 & 8 & 10 & 8 & 8 & 8 \\
\hline saritasignegmail.com & 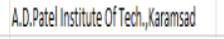 & 9 & 6 & 9 & 8 & 6 & 8 & 7 & 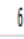 & 6 \\
\hline trijinysegmail.om & ADAN INSTTUTEOF NFRASTRUCTREEN & 10 & & 9 & 9 & 9 & 9 & 9 & 9 & 9 \\
\hline
\end{tabular}
similarity metric. [3]

Matrix factorization techniques for recommender systems the recommender system is based on one of two strategies. The external information is required for the system, so it needs to collect external information. The collection of external information is not easy. The content-based strategies require gathering external information that might not be available. [4] The collaborative filtering plays a vital role in various automatic recommendation systems and has been used in many online applications. Successful as they are, one limitation of most existing collaborative filtering algorithms is that they are static models in which relations are assumed to be fixed at different times. Learning from relational data has always been a central topic in the fields of data mining and machine learning.[4] 


\section{DATA ANALYSIS}

For a good and accurate result, we require a large dataset. The required data for this dataset is collected from various colleges and students from these colleges. We train our model based on possible ways:

\section{A. Last year cut-off}

\begin{tabular}{|c|c|c|c|c|c|c|}
\hline Course name & Inst_Name & Category & Subcat & Opening & osing & \\
\hline AUTOMOBILE ENGINEERING & A.D.Patel Institute Of Tech, Karamsad & OPEN & Others & 8586 & 35275 & 2018 \\
\hline AUTOMOBILE ENGINEERING & A.D.Patel Institute of Tech.,Karamsad & SEBC & Others & 37119 & 38197 & 2018 \\
\hline IUTOMOBILE ENGINEERING & A.D.Patel Institute of Tech.,Karamsad & ST & Others & 9548 & 39548 & 2018 \\
\hline AUTOMOBILE ENGINEERING & A.D.Patel Institute Of Tech.,Karamsad & OPEN & Other & 12430 & 33025 & 2019 \\
\hline AUTOMOBILE ENGINEERING & A.D.Patel Institute Of Tech.,Karamsad & GEN & Other & 650 & 18160 & 2017 \\
\hline AUTOMOBILE ENGINEERING & A.D.Patel Institute Of Tech.,Karamsad & SEBC & Other & 19611 & 33594 & 2017 \\
\hline AUTOMOBILE ENGINEERING & A.D.Patel Institute & ESM & Other & 31490 & 31490 & 2017 \\
\hline AUTOMOBILE ENGINEERING & A.D.Patel Institute Of Tech,,Karamsad & SC & Other & 34947 & 37317 & 2017 \\
\hline AUTOMOBILE ENGINEERING & A.D.Patel Institute Of Tech, Karamsad & GEN & Other & 10114 & 19318 & 2016 \\
\hline AUTOMOBILE ENGINEERING & A.D.Patel Institute Of Tech,,Karamsad & SC & Other & 30134 & 34611 & 2016 \\
\hline AUTOMOBILE ENGINEERING & A.D.Patel Institute Of Tech, Karamsad & ST & Other & 40947 & 40947 & 2016 \\
\hline UTOMOBILE ENGINEERING & A.D.Patel Institute Of Tech, Karamsad & SEBC & Other & 19476 & 32968 & 201 \\
\hline
\end{tabular}

Figure 1. Last year cut-off dataset

This dataset is built by collecting the last four years' vacant quota and four years cutoff form the ACPC website.

\section{B. User ratings}

This dataset is built by collecting reviews from the alumni and the current students of the college. Users who had given ratings to their respective colleges based on different parameters on the scale of 1-10. This dataset is used to generate recommendations for new users. We make students feedback from which consists of 10 attributes based on which questions are framed.

For example,

Figure 2. College Rating dataset

a) How the important college infrastructure is for you? (Rate it Accordingly)

b) How important Industry for you? (Rate it Accordingly)

c) How much college campus life important to you while selecting a college? (Rate it Accordingly)

d) How much placement activates is matter to you? (Rate it Accordingly)

Above mentioned dataset is collected from faculties and students of various engineering colleges. The fields of the dataset we have taken into consideration are Infrastructure, hostel facility, sports, library facility, college life, alumni support, culture event, security/discipline, academic and industry exposure. Using this data, we calculate overall weighted grade and further we use this to recommend the college.

\section{EXPERIMENT AND OBSERVANT}

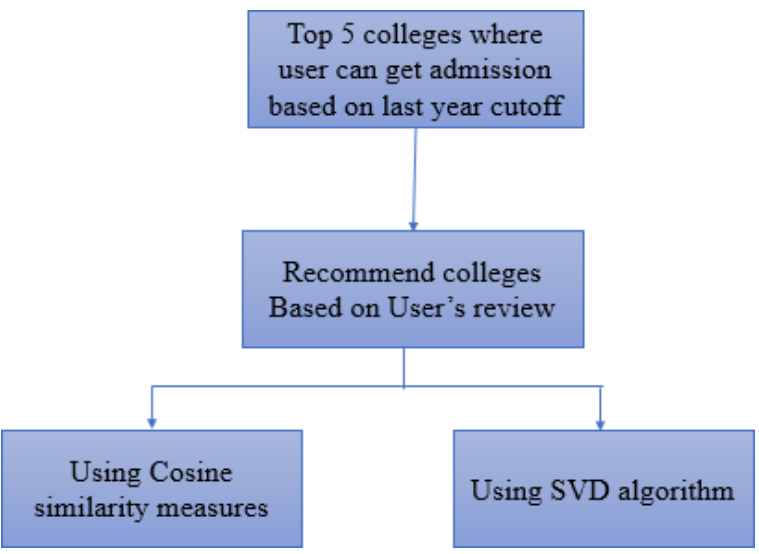

Figure 3. System Flow

Various technologies are used in the implementation as Tkinter mainly used for designing an interface. Python, Anaconda navigator, Jupyter Notebook, sklearn, and surprise packages are used to implement the recommendation system. our system Recommend based on:

\section{A. Based on merit rank}

We are going to recommend colleges as per student merit rank, branch, category using last four years cutoff shown in figure 3. that we collected from the official website for admissions in Gujarat. The user enters his merit rank, in which branch he wants to get admission and which category he belongs to. From all parameters and according to the current year our system will recommend the top 5 colleges list.

\section{ENTER YOUR MERIT NO:}

ENTER YOUR BRANCH:

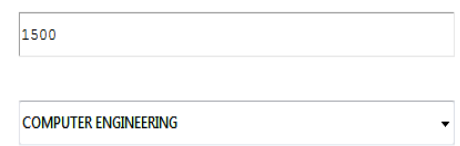

ENTER YEAR:

\section{ENTER YOUR CATEGORY:}
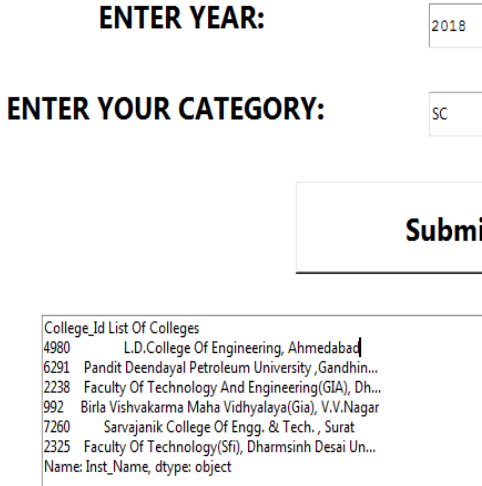

Figure 4. Top 5 college list based on cut-off

\section{B. Based on user's view (similarity measure)}

We collecting reviews from the alumni and the current students of the college. Users who had given ratings to their respective colleges based on different parameters on the scale of 1-10. we collect college rating based on 10 different parameters like infrastructure, hostel facility, sports, library facility, college life, alumni support, culture event, security/discipline, academic, and industry exposure then we calculate weighted mean value for that. for further processing, we took email_id as user_id and college name as collage_id then apply label encoder on that to convert a categorical value into a numeric value. 


\section{Recommender System for Engineering College}

we find similarities between colleges and then find cosine similarities matrix which gives similarities between college to college.

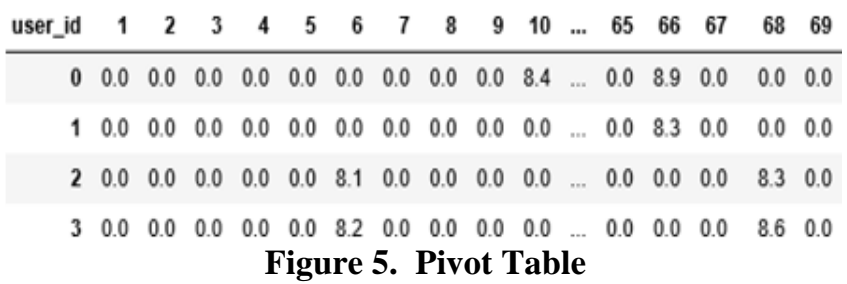

As shown in figure 5. the pivot table creates a matrix of users and college_ratings. we take user_id as column and college_id as row.it display grade value in each cell of college_id and user_id.

\begin{tabular}{|c|c|c|c|c|c|c|c|c|c|}
\hline 0 & & 1 & 2 & 3 & 5 & 6 & 7 & 8 & \\
\hline 0 & & 150.54433 & 0 & 0.225048 & 480.641077 & 70.159279 & 790.596306 & 60.16 & 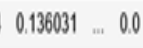 \\
\hline & & & 68 & 810 & 31 & 30.250014 & 85216 & 60.252523 & $07635 \ldots$. \\
\hline & & & 000.695336 & 360.680138 & 380.765996 & 60.658345 & 450.749962 & 20.659185 & $0.646807 \ldots$ \\
\hline & 0.46868 & 810.69533 & 360.00000 & 000.708165 & 0.600346 & 60 & $80 \quad 0.657561$ & 10 & $719049 \ldots$ \\
\hline & & & & & NJ & 1 & $00 / 460$ & 0.0 .182 & 0.791968 \\
\hline
\end{tabular}

Figure 6. Cosine Matrix

As shown in figure 6. It displays a similarity matrix between colleges.

\section{Enter Name of Collage $=$}

\section{Submit}

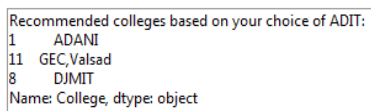

Figure 7. Top 5 college list based on user's review

As shown in figure 7. the user enters the college name and our system will recommend the top 5 colleges which are similar as per the user's review.

\section{Based on user's view (SVD algorithm)}

For further processing, we took email_id as user_id and college name as collage_id then apply label encoder on that to convert a categorical value into a numeric value. While finding similarity measures, biased user's review can also be calculated. Another approach which can resolve this is SVD algorithm to calculate matrix factorization. We have recommended colleges based on algorithm.

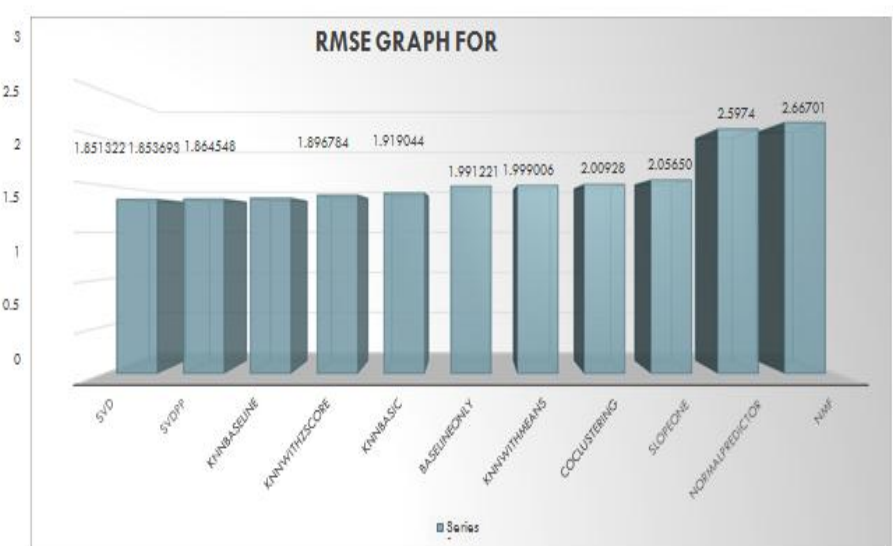

Figure 8. RMSE Graph

Comparison of Result

\begin{tabular}{|l|l|l|}
\hline College Name & Based on similarity measure & Based on SVD algorithm \\
\hline ADIT & ADANI & \\
& MBICT & VVP \\
& GCET & DPASNT \\
& LDCE & GCET \\
& SNPIT & SNPIT \\
\hline
\end{tabular}

\section{CONCLUSION}

This application helps students to predict the institute where one can get admission as per the previous year cut off. Further, he can have a recommendation of similar colleges as per the user's review. So, one can decide which college is better for her/his education. It is a recommender system that will use past data and suggest the most appropriate college. It'll take students' information like merit rank and provide a list of colleges. It'll give them all the data regarding colleges like infrastructure, hostel, placement, etc and colleges list that is best suitable for them. The system can also be used by parents to search for the better choices available for their ward. The system can also be used by any person from engineering and non-engineering backgrounds to guide the students and their knowledge too.

The system is capable to recommend the colleges based on the user's review and is also capable to give the colleges based on merit numbers from previous year cut-off. This can be improved by collecting more records from various users. One can also improve and test the result of a recommendation by applying a different model-based algorithm.

\section{REFERENCES}

1. A User-Friendly College Recommending System Using User-Profiling and Matrix Factorization Technique by Sheetal Grease, Varsha Powar and Debajyoti Mukhopadhyay Published at May 2017 IEEE https://www.researchgate.net/publication/322006324.

2. Friendbook: A Semantic-Based Friend Recommendation System for Social Networks by Zhibo Wang, Jilongliao, Quimg Cao, Hairong Qi, and Zhi Wang Published at 2017 IEEE. 
3. Graduate School Recommendation System: Assisting Admission Seekers to Apply for Graduate Studies in Appropriate Graduate Schools by M. Hasan, S. Ahmed, D.M. Abdullah, and M.S. Rahman, 2016 IEEE.

4. Collage Recommendation System for Admission by Deokatemonali, Gholave Dhanashri, Jarad Dipali, Khomane Tejaswini at 2018.

\section{AUTHORS PROFILE}

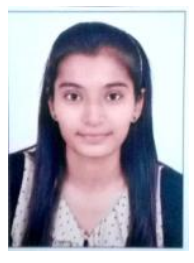

Meshwa Mistry, Final year student of the Computer Engineering Department is associated with G H Patel College of Engineering \& Technology, Vallabh Vidyanagar.She completed diploma from B \& B Institute of Technology.Currently working project is Recommendation System for Engineering College. Furthermore, she has also proved her competence by participating and winning in several technical competition in the field of programming. Moreover, she has contributed to many projects in her undergraduate study including "carpooling system", "Hospital management". Besides, her area of interest includes Big data, Databases and knowledge-bases, Machine Learning and Data Science.

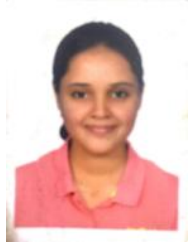

Vidhi Suthar, Final year student of the Computer Engineering Department is associated with G H Patel College of Engineering \& Technology, Vallabh Vidyanagar.She completed diploma from B \& B Institute of Technology.Currently working project is Recommendation System for Engineering College. Furthermore, she has also proved her competence by participating and winning in several technical competition in the field of programming. Moreover, she has contributed to many projects in her undergraduate study including "Food corporation of India", "Hospital management". Besides, her area of interest includes Big data,python,Machine Learning and Deep learning.

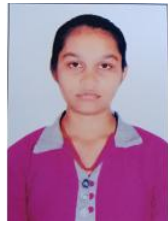

KaPatel Shruti Final year student of the ComputerEngineering Department is associated with $\mathrm{G} \mathrm{H}$ Patel College of Engineering \& Technology, Vallabh Vidyanagar.She completed diploma from B \& B Institute of Technology.Currently working project is Recommendation System for Engineering College. Furthermore, she has also proved her competence by participating and winning in several technical competition in the field of programming. Moreover, she has contributed to many projects in her undergraduate study including "Food corporation of India", "Hospital management". Besides, her area of interest includes Big data,python,Machine Learning and PHP, MySQL.

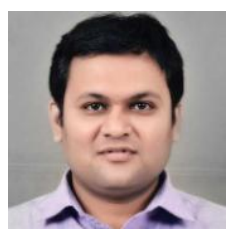

Priyang Bhatt (Assistant Professor) joined GCET in 2007. He pursued his B.E in Computer Engineering from Charotar Institute of Technology (Now CHARUSAT), Changa, Gujarat University and M.E. in Computer Engineering from Birla Vishwakarma Mahavidyalaya, Sardar Patel University. $\mathrm{He}$ is currently pursuing his Ph.D. from Guja Technological University in the area of Securing Internet of Things (IoT). He is CCNA, RHCE and RHCSA certified. He has developed and published an Android app for first-year students with the name "CPU EXAM PREP" on the Google Play Store. he has conducted the number of workshops and delivered expert talks on the area of Machine 